\title{
Correction to: Prevalence and risk factors of sexual dysfunction in patients with inflammatory bowel disease: systematic review and meta-analysis
}

\author{
Jinzhi Zhang ${ }^{1} \cdot$ Shi Wei ${ }^{2} \cdot$ Qishan Zeng ${ }^{3} \cdot$ Xinyao Wu ${ }^{1} \cdot$ Huatian Gan ${ }^{1,3}$ \\ Published online: 19 July 2021 \\ ๑) Springer-Verlag GmbH Germany, part of Springer Nature 2021
}

\section{Correction to: International Journal of Colorectal Disease https://doi.org/10.1007/s00384-021-03958-y}

In the original published version of this article, the orders of the authors Jinzhi Zhang and Shi Wei in the authorship section were interchanged. Jinzhi Zhang should have been the first author and Shi Wei as second author. Both authors have contributed equally to this work.

The original article has been corrected.

The original article can be found online at https://doi.org/10.1007/ s00384-021-03958-y.

Huatian Gan

1457915156@qq.com; ganhuatian@hotmail.com

Jinzhi Zhang

zhangjzh96@126.com

Shi Wei

zhangjzh96@163.com

Qishan Zeng

15928646490@163.com

Xinyao Wu

1299619527@qq.com

1 Department of Geriatrics and National Clincal Research Center for Geriatrics, West China Hospital, Sichuan
University, No. 20, Section 3, South Renmin Road, Chengdu 610041, Sichuan, People's Republic of China

2 Department of Obstetrics and Gynecology, West China Second University Hospital, Sichuan University, No. 37 Guoxue Alley, Chengdu 610041, Sichuan, People's Republic of China

3 Lab of Inflammatory Bowel Disease, Center for Inflammatory Bowel Disease, Clinical Institute of Inflammation and Immunology, Frontiers Science Center for Disease-Related Molecular Network, West China Hospital, Sichuan University, No. 37 Guoxue Alley, Chengdu 610041, Sichuan, People's Republic of China 